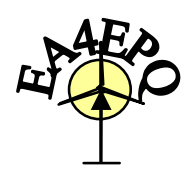

International Conference on Renewable Energies and Power Quality (ICREPQ’09)

Valencia (Spain), 15th to 17th April, 2009

\title{
Permanent Switchboard Monitoring using Embedded Web Server
}

\author{
F. Fioretti ${ }^{1}$, S. Pasqualini ${ }^{1}$, A. Andreoli ${ }^{1}$ and P. Pierleoni ${ }^{1}$ \\ ${ }^{1}$ Dipartimento di Ingegneria Biomedica, Elettronica e Telecomunicazioni \\ Università Politecnica delle Marche \\ Via Brecce Bianche 1, 60131 Ancona (Italy) \\ Phone/Fax number:+0039 071 2204847, e-mail: f.fioretti@univpm.it, s.pasqualini@univpm.it, a.andreoli@univpm.it, \\ p.pierleoni@univpm.it
}

\begin{abstract}
The proposed paper discusses the realization of a system prototype able to perform a permanent monitoring of a switchboard in order to allow the manufacturer the complete management of all the measurable quantities in the switchboard, specially the sensed voltage for each line. The main purpose is to prevent any fault that can affect the switchboard and provide an instant alarming if anomalous values are revealed. The significant aspect of our prototype is the internetworking between several standard network protocols. In fact, the acquisition network sends the acquired data using the EIA 485 serial protocol. These data are then collected into an embedded web server ad-hoc developed. The server can be connected to the Internet in a wired and/or wireless way using the IEEE 802.3 (Ethernet) and the IEEE 802.11 (Wi-Fi) protocols. Finally, the web server uses standard TCP/IP protocols, such as HTTP to manage the web pages and SMTP to dispatch automatic alarm notifications via e-mails.
\end{abstract}

\section{Key words}

EIA 485, Embedded Web Server, Power Quality, Switchboard monitoring, TCP/IP, Wi-Fi.

\section{Introduction}

Electrical systems are necessary in each kind of building such as homes, factories, hospitals and so on. In many cases electrical systems manage critical applications, like freezer temperature, incubator internal temperature and so on. All these systems contain devices that are sensitive to voltage variations, current deviations and to all the power quality measurable quantities [1]. Power quality issues interest both national supplier and customers as explained in [2] and [3]. In addition to proposed architectures and systems also network simulation of power quality systems are realized [4]. In all these documents we can find that one of the possible solution for power quality data acquisition is based on permanent monitoring systems: for our purpose we refer to the switchboards components. Benefits of such systems are numerous, first the opportunity to prevent any fault that could affect the switchboard. In fact, these systems are able to detect malfunctions due to several causes. For example, a faulty switch can provide a lower voltage to an electrical engine: the switch is open, but the engine does not work properly.

Moreover permanent monitoring architectures can be realized in several ways. Many companies develop a proper system based on proprietary protocols, or on BAC (Building, Automation and Control) architectures, such as KNX [5] or LonWorks [6]. These systems are reliable, but they require a protocol license fee, and the interoperability between devices produced by different companies is not guaranteed. Therefore, the monitoring system can not be easily extended to new devices installation.

Permanent monitoring systems can also be realized using servers, both web and database servers, able to store data and to be easily accessed by the Internet network. Several works involve this kind of devices [7][8]. Also another monitoring system based applied to biomedical subject is described in [9]. In this work we use embedded web servers to realize an Internet based data acquisition system [10]. These kind of devices are very useful to store a limited amount of data, they are programmable and compatible with all the TCP/IP protocols (e.g. HTTP for web servers and SMTP for e-mails delivery).

If the embedded web server is used to store the data and to publish them on the Internet, the acquisition network has the role of a transducer between the devices and the web server. For our purpose we chose a serial network. In literature several serial networks are proposed, some using optical fiber as physical medium [11]. Also integration between serial networks and PLC (Programmable Logical Controllers) are proposed [12].

Novelty of the proposed system is the intensive employment of standard communication protocols. In fact, we realized an acquisition network able to measure the voltage of the switchboard that uses a standard serial protocol (EIA 485) to transmit data to an embedded web 
server. This server complies with the TCP/IP standards in order to publish the previously acquired data and to alarm notification send e-mails. The device implementing the web server can access the physical medium layer by the IEEE 802.3 and IEEE 802.11 standards, so both a wired and a wireless connection can be realized. Moreover, an ubiquitous control is possible thanks to the use of the TCP/IP standard protocols that are available in a wide range of applications installed on several kind of devices such as notebooks, PDAs, smart-phones, etc. In fact, using one of the existing web browsers, the switchboard status can be monitored by a remote user or the manufacturer. This allows an instant alarm notification and a fault recognition; moreover, using more complex circuits it is possible to act a remote control on the same switchboard.

\section{Overall Prototype Architecture}

The proposed monitoring system complies the architecture depicted in Fig. 1.

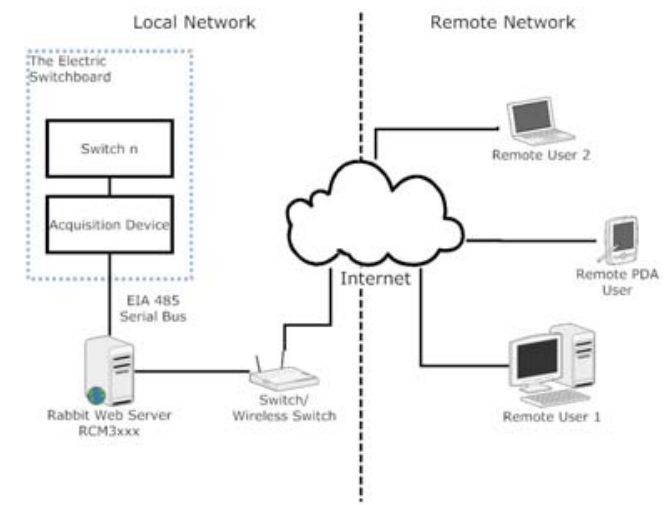

Fig. 1. Overall architecture for the switchboard monitoring prototype

As shown in the figure, the overall architecture can be split in two main parts: a local network and a remote one. The local network allows the acquisition of the sensed voltage from each switch that compose the switchboard. Thanks to this network we can evaluate one of the major Power Quality quantities: the voltage deviations. Voltage variations can affect both three-phases and single phase devices. The acquisition device, that acts as a transducer, is connected to the switch and it produces an analog voltage proportional to the voltage phase. Then this voltage is sent and it is converted into a digital signal in order to be encapsulated into an EIA 485 frame. Using this serial bus, the transducer is connected to an embedded web server implemented using the RCMs (Rabbit Core Modules) produced by Rabbit Semiconductors, whose description will follow in the next sections. The embedded web server is connected to the Internet by a switch or an access point depending on the usage of a wired or wireless connection.

In the remote network each user can perform a permanent monitoring using one of the available web browsers, such as Internet Explorer, Mozilla Firefox, and so on. The monitoring system is based on HTML pages that are dynamically updated by a CGI (Common Gateway Interface) function. Finally a remote user can also receive alarm e-mails in order to be aware about any troubles or malfunctions in the switchboard.

\section{Acquisition Network}

This section covers the acquisition network implementation, which schema is shown in Fig. 2. This network acts as a transducer between all the devices composing the switchboard and the embedded web server. The first issue that we consider refers the voltage conversion: for each line of any switchboard device we can measure a low voltage up to about $400 \mathrm{~V}$. So we have to reduce this value in order to keep it compatible with the maximum voltage allowed by the RCMs. This conversion is achieved by a device provided by Phoenix Contact. In particular, these devices can handle the three lines, producing a scaled DC voltage proportional to the real one. Then, the produced voltage must be converted into a digital value using an Analog to Digital Converter.

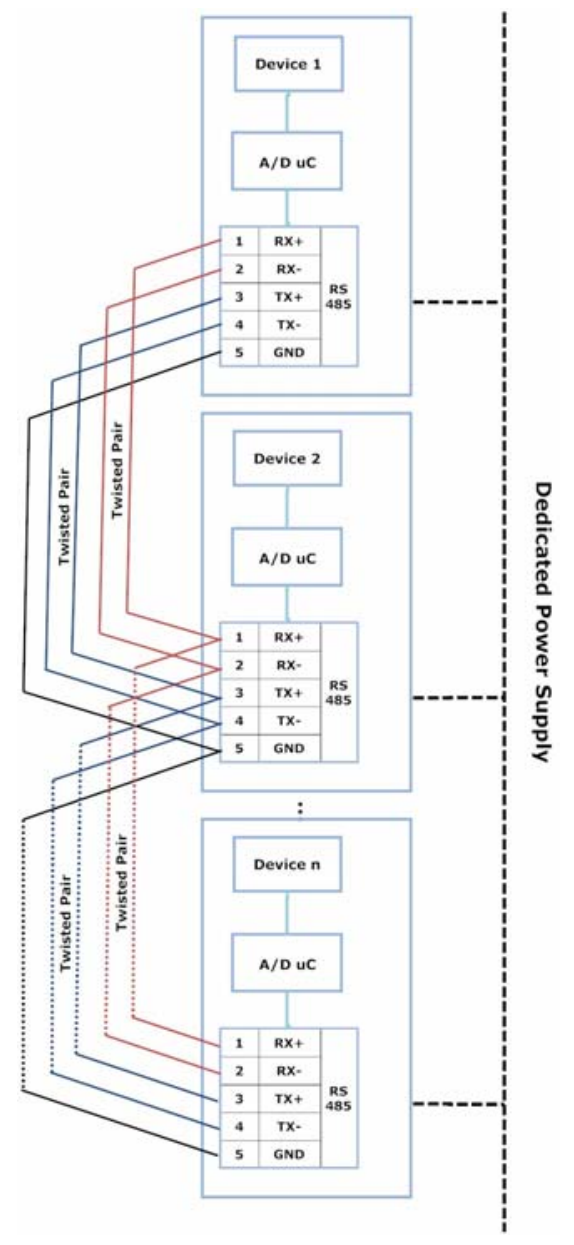

Fig. 2. Acquisition Serial Network

As known, many components compose the switchboard, so we have to use a suitable network topology. For our purposes we chose a serial network based on the EIA 485 protocol, as explained in the figure. This protocol allows many topologies implementation, but the daisy chain connection is the preferred. Also, we retain it the best choice. This because in all the switchboards assemblages 
there are many wires to be connected and other topologies, first a star topology, will produce an increase of the wires number. Instead, the daisy chain topology allows to use a reduced number of wires, where the output of a device is connected to the input of the following devices. Moreover, the daisy chain connection allows an easy integration of new devices: it is only required to break the chain in order to add the new device and connect it with the neighbours devices.

Finally, all the RCMs are able to manage EIA 485 frames.

\section{Embedded Web Server}

This section explains the embedded web server used for the proposed implementation. After a brief description of the device and its input/output ports, we focus on the programming issues, the supported TCP/IP protocols and the code description of the prototype.

\section{A. Implementation}

The web server was realized using the RCM3xxx family. The Ethernet module is depicted in Fig. 3. The RCM3xxx are based on a 8-bit Rabbit processor with $20 \mathrm{MHz}$ clock. Depending on the specific type of the modules, also processor with a $44.2 \mathrm{MHz}$ clock can be used. RCMs are tolerant to a wide number of analog lines that arrive to $5 \mathrm{~V}$. They have 3.3V CMOS compatible serial ports, too. So, these modules can be easily connected to SPI (Serial Peripheral Interface) and I2C (Inter Integrated Circuit) devices. Referring to the memories, the RCMs have a 512kB flash memory, 512kB program execution SRAM, $512 \mathrm{kB}$ data SRAM. The operating code data can be stored permanently into the flash memory using a Dynamic C FAT (File Allocation Table) file system. Since a so small flash can be a lack in a web server implementation, it is possible to extend the amount of memory using a serial flash that can be bought separately. The serial flash can have a size of $4 \mathrm{MB}$ or $8 \mathrm{MB}$.

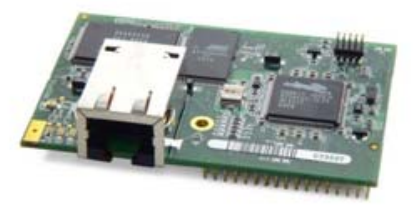

Fig. 3. Rabbit Core Module 3xxx

The RCMs programming language is the Dynamic C, very close to the ANSI $C$ [13]. The operating code written is then stored into the flash and it is executed when the module is powered on. RCMs have not an Operating System: in this way the boot time is very short because it has the only commit of loading the operating code. Finally, the operating code sets the file system, stores external files (web pages, images, textual files and so on) and manage the network protocols and the network access. In fact, both a wired and a wireless solution are used in our application, using IEEE 802.3
(Ethernet) and IEEE 802.11 (Wi-Fi) as data-link layers. Referring to the higher levels of the TCP/IP stacks, the Dynamic C manage both TCP and UDP connections and a wide range of application level protocols, such as HTTP, SMTP, and FTP.

More information about the module description, including the processor and the $\mathrm{I} / \mathrm{O}$ of the development board can be found in [14].

\section{B. Web Server Pages}

This section addresses the web pages design. The proposed prototype has embedded server pages that are stored into the flash of the embedded web server. The employed web pages are both static and dynamic. Static HTML pages are used in order to view contents that do not change, providing only an informational view, while dynamic pages are used to manage data that can vary. The dynamic update is performed using CGI functions that will be explained in next section.

Specially, the developed prototype includes the following pages:
1) Login form
2) Home Page
3) Switchboard Information and Configuration
4) On line monitoring
5) Event Log repository

The home page must contain all the links to the other web pages stored into the embedded web server. The home page can only be accessed after an authorization procedure using the HTTP protocol.

The switchboard Information and Configuration contains all the necessary information about the switchboard, such as layout dimensions, electrical schemas, and so on. All these data are collected into a static HTML page that will be uploaded during the programming stage.

Relating to the on line monitoring, a dynamic page must be created in order to have an instant view on the load measured on each switchboard component. To facilitate the analysis of the acquired voltage, all the malfunctioning must be detected in a very easy way: this is achieved by the usage of a color legend. Each color is associated to a specific error: connection failure, voltage deviation, and not available data.

Finally, the log event page is composed by a textual file that is stored permanently into the embedded flash. This file contains all the information related to any malfunctioning. It reports the ID of the switchboard device, the timestamp of the event notification and the sensed voltage. This file is very useful to detect also the duration of each event in order to provide the right classification among the power quality quantities.

Other settings can be made by a web form that can be accessed only by the manufacturer. It contains several settings: the automatic refresh time (default 1 second), 
the switchboard information page upload and a configuration file upload that contains all the ID of the switchboard devices and specify if the automatic email dispatch is required in order to perform an instant alarming.

\section{Dynamic Page Creation}

As explained in the previous section, the proposed monitoring system is composed both of static and dynamic pages. In this section we cover the issues about the dynamic pages construction: basically we use a CGI function. The CGI functions allow the interfacing of external application into a web server. In this way it is possible to integrate other source code into an HTML page. For our purpose we wrote a CGI function as a Dynamic C code.

Before starting with the CGI acquisition function description, we will consider the $\mathrm{C}$ structure used to model the switch state, listed in the follow:
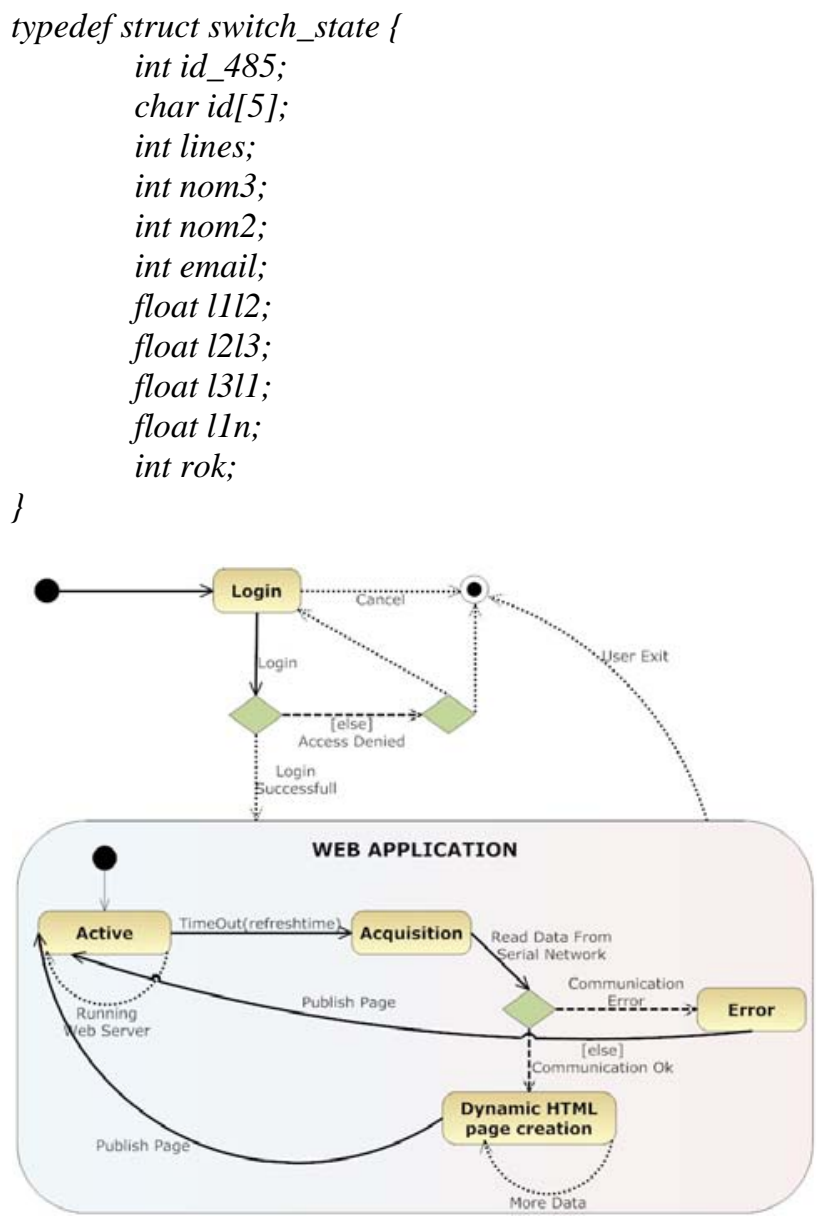

Fig. 4. UML state diagram of the on line monitoring procedure

Looking at the Dynamic C code, we identify each switch by two variables: the EIA 485 identifier and a mnemonic ID that is the same used during the switchboard assembling procedure. For any kind of switch we have to identify the number of lines where it acts and for each couple of lines we have to specify the nominal voltages. Also each voltage is stored into the variables $1112,12 l 3$,
$1311,11 n$. Moreover, additional settings are stored into the email variable that will be set to one if an automatic email dispatch will be required and the rok variable will be set to one if the reading stage will be successful. Finally, the switchboard is composed by one ore more of these structures.

To describe the CGI function, we will use the UML (Unified Modeling Language) to represent the state diagram. Since it is not possible to describe the only CGI function without taking into account the overall prototype, in Fig. 4 the overall state chart is represented.

As explained in the same figure, the user must be authenticated in order to access the web application. This procedure is shown in the top of the figure, where starting from the default connection, we step into the "Login state". From this particular state there are two events: the access denied causes the exit from the web application or a redirect into the "Login" state, while the successful login procedure allows an user to access the web application. We want to underline that we set the USE_HTTP_DIGEST_AUTHENTICATION macro to 1 in order to enable the digest authentication to the embedded web server. This kind of authentication provides more security relating to the basic authentication, where both username and password are sent as plain text. In fact, the digest authentication applies the MD5 cryptographic hashing to prevent any cryptanalysis attacks.

The web application state chart, implemented by a CGI function and depicted in the bottom of the figure, explains how the web application works. The default transition leads to the "Active" state. The application remains in the same state and it works as a common web server, allowing the access to all the available web pages. When the refresh time is elapsed, then a time-out event occurs causing the transition to the "Acquisition” state. In this state the application is ready to read the data coming from the serial acquisition network. So, after the Read Data event two possible states can be reached: if a communication error occurs, then the end state is "Error" that produce a web page that report this error. If no communication errors are revealed, we reach the "Dynamic HTML page creation" state. The publish page event allow the dynamic creation, where all the retrieved data are used to create an HTML page, following what was previously written into the switch_state structures.

\section{Test on the Prototype}

TABLE I. - Switchboard Configuration

\begin{tabular}{|l|c|}
\hline \multicolumn{1}{|c|}{ ID } & DESCRIPTION \\
\hline QM1 & 3P Switch \\
\hline K1 & 3P+N Switch \\
\hline Q2 & 2P Switch \\
\hline Q3 & 3P+N Switch \\
\hline Q4 & 3P+N Switch \\
\hline
\end{tabular}


This section covers the realized prototype test. So, using the screenshots coming from the developed application we will show how it is possible to use the proposed data acquisition system for a switchboard whose configuration is in Table I.

As explained we have to reach the embedded web server by typing its address into the web browser (for example we tested the prototype in a LAN, where the server has the 192.168.48.74 address). This produce the login form of Fig. 5. During the programming stage we set the credentials of the authorized users.

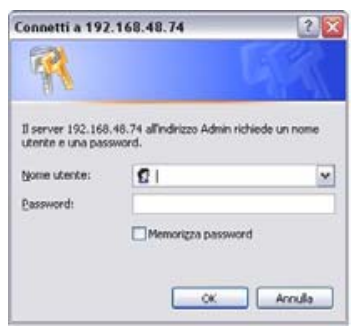

Fig. 5. Login Page

After a successful login it is possible to reach the home page of the web application (see Fig. 6). Basically it addresses to all the other contents of the application.

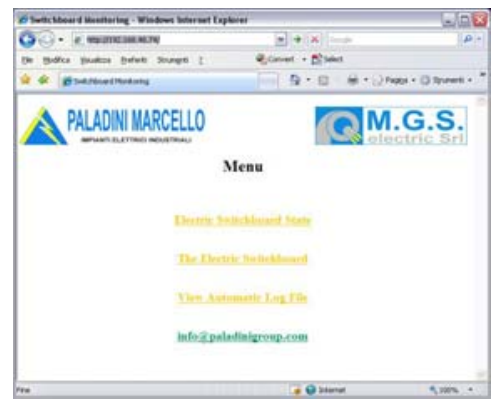

Fig. 6. Prototype Home Page

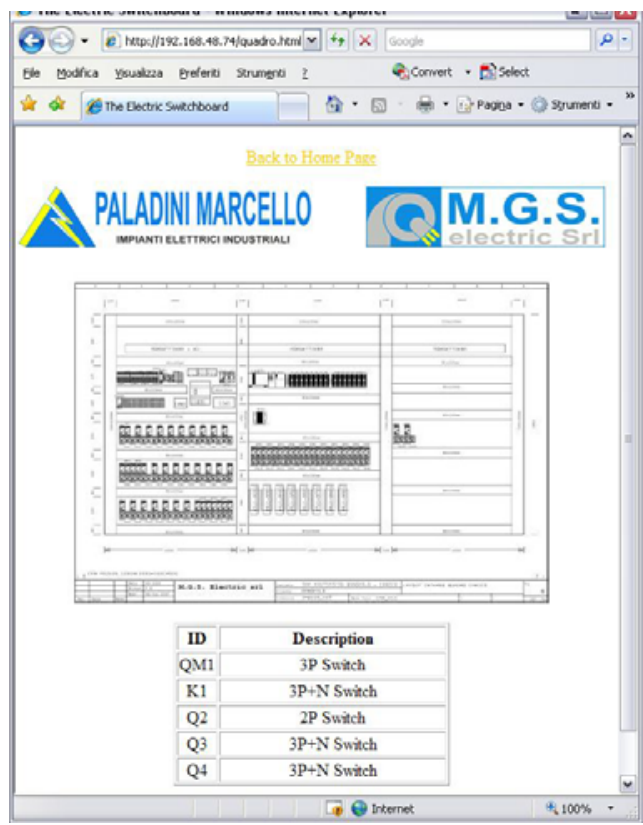

Fig. 7. Switchboard Information for the prototype

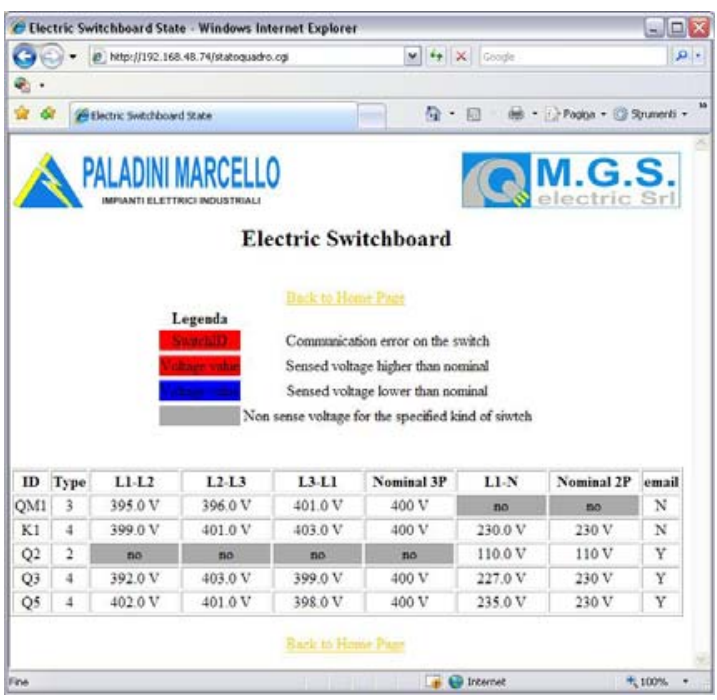

Fig. 8. Example of an on-line monitoring page

The static web page relating to the switchboard information is depicted in Fig. 7. It only shows the switchboard layout and the table containing all the connected devices including their identification. Finally, the Fig. 8 shows an example of the dynamic page created for the on-line monitoring.

\section{Comments on the Prototype}

The proposed architecture has a lot of benefits. First the usage of standard protocols both for the acquisition network and for the remote monitoring. Moreover the Internet connection can be realized using both Ethernet and Wi-Fi data link protocols. Also embedded web servers guarantee an high robustness in terms of boot time and they can work also without any power supply thanks to the integrated backup battery. The prototype can be easily extended to more devices and the web server settings can be changed by the remote authorized users, too.

This prototype can be developed using other technologies. Regarding the security issue, the prototype take into account only a cypher authentication. So, all the data can be protected using a SSL (Secure Sockets Layer) communication. This protocol allows security and data integrity over the Internet and it is included into the RCMs supported protocols.

Referring to the user interface, it is possible to develop a more complete GUI (Graphical User Interface) providing an ad-hoc application instead of the HTML pages. A such application can be realized using an XML for Power Quality Data [15] or the PQDIF (Power Quality Data Interchange Format). Using this languages, the RCM can be used as a network server, able to generate strings that will be sent to remote user. The remote user has to execute the application in order to receive these words and parse them. Moreover, a more powerful GUI can be used as a more efficient monitoring and diagnosis system because the acquired voltages can be processed in order 
to obtain other power quality quantities, such as the harmonics.

\section{Conclusion}

The proposed paper shows a prototype of a permanent monitoring system applied to the management of a switchboard. This prototype is based on standard communication protocols: the EIA 485 to transmit data from the acquisition device to the embedded web server and the TCP/IP protocols (HTTP and SMTP) for the authenticated users remote access and e-mail reception. In this way an high internetworking is achieved by the concurrent use of TCP/IP and serial protocols. Communication between remote users and the monitoring system can be performed both in a wired and a wireless manner.

We can assert that the proposed prototype can be very useful in Power Quality contests because a permanent supervising of an electric switchboard allows a correct usage of each switch, in a such way any anomalous behaviour can be revealed. This grants the manufacturer an immediate action in order to repair the faults that can occur and a consequently longer life cycle of each switch. The proposed system can also be extended in order to allow the full control of the switchboard. Moreover, further development can involve this kind of prototype, first a secure channel creation that will lead to the data integrity. Also an integration with the PQDIF or a XML language will permit a more powerful user interface without any increase in the server complexity. In this way it is possible to consider other Power Quality quantities that can be evaluated thanks to post acquisition elaborations. So, the prototype will be a valid diagnosis instrument.

Finally, the prototype is based on open and standard protocols. This key feature allows low cost realization because no royalty must be paid due to the use proprietary protocols. The architecture can also be easily extended for an higher number of devices.

\section{Acknowledgement}

The authors thank the "Paladini Marcello I.E.I" and "M.G.S. Electric s.r.l” for the technical support during the prototype development.

\section{References}

[1] J. Stones and A. Collinson, "Power quality", Power Engineering Journal [see also Power Engineer], vol. 15, pp. 58-64, Apr 2001.

[2] P. Lim, "Power quality program "from source to socket"“, Power System Technology, 2004.
PowerCon 2004. 2004 International Conference on, vol. 2, pp. 1920-1924 Vol.2, Nov. 2004.

[3] E. Mertens, L. Dias, E. Fernandes, B. Bonatto, J. Abreu, and H. Arango, "Evaluation and trends of power quality indices in distribution system", Electrical Power Quality and Utilisation, 2007. EPQU 2007. 9th International Conference on, pp. 1-6, Oct. 2007.

[4] Y. Bi, J. Zhao, and D. Zhang, "Research on Power Communication Network and Power Quality Monitoring Using OPNET”, Industrial Electronics and Applications, 2007. ICIEA 2007. 2nd IEEE Conference on, pp. 507-511, May 2007.

[5] Konnex Association, "KNX Specifications Version 1.1.” Digiem, Belgium, 2004.

[6] ANSI/EIA, ANSI/EIA 709.1-A-1999, “Lontalk Protocol”, 1999.

[7] I.-Y. Chung, D.-J. Won, J.-M. Kim, S.-J. Ahn, S.-I. Moon, J.-C. Seo, and J.-W. Choe, "Development of power quality diagnosis system for power quality improvement”, Power Engineering Society General Meeting, 2003, IEEE, vol. 2, pp. -1261 Vol. 2, July 2003.

[8] R.-C. Leou, W.-R. Tsai, and Y.-N. Chang, “A power quality monitoring system based on J2EE architecture", TENCON 2004. 2004 IEEE Region 10 Conference, vol. C, pp. 291-294 Vol. 3, Nov. 2004.

[9] Y. Li, S. Wu, J. Li, and Y. Bai, "The ECG TeleMonitor Based on Embedded Web Server", Bioinformatics and Biomedical Engineering, 2007. ICBBE 2007. The 1st International Conference on, pp. 752-755, July 2007.

[10] I. Klimchynski, "Extensible embedded web server architecture for Internet-based data acquisition and control”, Sensors Journal, IEEE, vol. 6, pp. 804811, June 2006.

[11] R. Skegg, "A high speed fiber optic serial networking system for data acquisition and control”, Nuclear Science, IEEE Transactions on, vol. 37, pp. 293-297, Apr 1990.

[12] M. Cho and J. Hwang, "Development of data acquisition and load control system by programmable logic controller for high voltage load customer", Power System Technology, 1998. Proceedings. POWERCON '98. 1998 International Conference on, vol. 1, pp. 275-279 vol.1, Aug 1998.

[13] Z-World inc, “Dynamic C User’s manual”, 2000.

[14] Rabbit Semiconductor, "RabbitCore RCM3xxx User's Manual”, 2005.

[15] S. Chen and X. Wang, "Power quality XML markup language for enhancing the sharing of power quality data”, Power Engineering Society General Meeting, 2003, IEEE, vol. 3, pp. -1570 Vol. 3, July 2003. 